\title{
Theta burst stimulation improves overt visual search in spatial neglect independently of attentional load
}

Dario Cazzoli a,b, Clive R. Rosenthal ${ }^{\text {a }}$, Christopher Kennard ${ }^{\text {a }}$, Giuseppe A. Zito $^{c}$, Simone Hopfner ${ }^{d}$, René M. Müri ${ }^{\mathrm{d}, \mathrm{e}}$, Thomas Nyffeler ${ }^{\mathrm{d}, \mathrm{f},{ }^{*}}$

${ }^{a}$ Nuffield Department of Clinical Neurosciences, University of Oxford, John Radcliffe Hospital, OX3 9DU Oxford, United Kingdom; ${ }^{b}$ University Hospital of Old Age Psychiatry, University of Bern, Murtenstrasse 21, 3010 Bern, Switzerland; ${ }^{c}$ ARTORG Center for Biomedical Engineering Research, University of Bern, Murtenstrasse 50, 3010 Bern, Switzerland; ${ }^{d}$ Perception and Eye Movement Laboratory, Departments of Neurology and Clinical Research, Inselspital, Bern University Hospital, and University of Bern, Freiburgstrasse 10, 3010 Bern, Switzerland; ${ }^{e}$ Division of Cognitive and Restorative Neurology, Department of Neurology, Inselspital, Bern University Hospital, 3010 Bern, Switzerland; ${ }^{f}$ Center of Neurology and Neurorehabilitation, Luzerner Kantonsspital, 6000 Luzern 16, Switzerland.

\footnotetext{
* Corresponding author: Prof. Dr. Thomas Nyffeler; Perception and Eye Movement Laboratory; Department of Neurology; Department of Clinical Research; Inselspital, Bern University Hospital, and University of Bern; Freiburgstrasse 10; 3010 Bern; Switzerland; thomas.nyffeler@luks.ch; Fax: +41/(0)41 20524 41; Tel.: +41/(0)41 2055686
} 


\section{Abstract}

Visual neglect is considerably exacerbated by increases in visual attentional load. These detrimental effects of attentional load are hypothesised to be dependent on an interplay between dysfunctional inter-hemispheric inhibitory dynamics and load-related modulation of activity in cortical areas such as the posterior parietal cortex (PPC).

Continuous Theta Burst Stimulation (cTBS) over the contralesional PPC reduces neglect severity. It is unknown, however, whether such positive effects also operate in the presence of the detrimental effects of heightened attentional load.

Here, we examined the effects of cTBS on neglect severity in overt visual search (i.e., with eye movements), as a function of high and low visual attentional load conditions. Performance was assessed on the basis of target detection rates and eye movements, in a computerised visual search task and in two paper-pencil tasks. cTBS significantly ameliorated target detection performance, independently of attentional load. These ameliorative effects were significantly larger in the high than the low load condition, thereby equating target detection across both conditions. Eye movement analyses revealed that the improvements were mediated by a redeployment of visual fixations to the contralesional visual field.

These findings represent a substantive advance, because cTBS led to an unprecedented amelioration of overt search efficiency that was independent of visual attentional load.

Keywords: Eye movements; Hemispatial neglect; Repetitive transcranial magnetic stimulation (rTMS); Stroke; Visual attention. 


\section{Introduction}

Visual neglect is defined as the failure to orient, attend, and respond towards the contralesional side of visual space (Heilman, Watson, \& Valenstein, 1993). Visual neglect is most commonly associated with the incidence of a lesion within an extended network of cortical and subcortical areas centred in the right hemisphere (Corbetta \& Shulman, 2011). Critical cortical areas include the posterior and inferior parietal lobe (Mort et al., 2003), the superior temporal lobe (Karnath, Fruhmann Berger, Küker, \& Rorden, 2004), and the inferior frontal lobe (Husain \& Kennard, 1996), whereas the main subcortical regions implicated in neglect include the pulvinar nucleus of the thalamus, the putamen, and the caudate nucleus (Karnath, Himmelbach, \& Rorden, 2002). Moreover, disconnections of white matter fibre tracts also play an important role (Doricchi, Thiebaut de Schotten, Tomaiuolo, \& Bartolomeo, 2008), such as the superior longitudinal fasciculus, the inferior occipito-frontal fasciculus, and the superior occipito-frontal fasciculus (Karnath, Rorden, \& Ticini, 2009; Shinoura et al., 2009).

A hallmark of visual neglect is that its' severity is significantly exacerbated by heightened visual attentional load, such as when discriminating visual targets from an increasing number of distracters (Bonato, 2012; Sarri, Greenwood, Kalra, \& Driver, 2009). For instance, the same patient may perform within normal range in tests with low visual attentional load, but may show significant signs of visual neglect when assessed with tests with high visual attentional load. 
There are two main reasons to predict that the modulation of activity in the posterior parietal cortex (PPC) is key to addressing the load-related modulation of neglect severity. First, the attentional networks of the left and the right hemisphere are centred around the parietal cortices, and compete to direct visual attention towards the contralateral side of space, thereby inhibiting each other via transcallosal connections (Koch et al., 2011). Lesions within the righthemispheric attentional network lead to deficient inhibition towards the intact, contralateral left-hemispheric attentional network, and thus to pathological hyperexcitability (Baldassarre et al., 2014; Corbetta, Kincade, Lewis, Snyder, \& Sapir, 2005; He et al., 2007). The degree of hyperexcitability in the left, intact PPC has been shown to correlate with the degree of ipsilesional bias in the deployment of visual attention (Koch et al., 2008); namely, the greater the hyperexcitability in this area, the greater the neglect severity. Second, in healthy subjects, a heightened visual attentional load leads to a bilateral increase in neural activity within the attentional networks, including both left- and righthemispheric PPCs (Schwartz et al., 2005). Moreover, the strongest, linear increase in neural activity with increasing visual attentional load occurs in both the left and the right PPCs (Jovicich et al., 2001). Hence, in patients with leftsided visual neglect due to a right-hemispheric lesion, a load-related increase in neural activity may occur only in the left, intact PPC. This would increase the existing hyperexcitability even further, and trigger a greater imbalance in interhemispheric inhibition, resulting in an exacerbation of visual neglect severity. One of the key approaches to tackling this pathological hyperexcitability in the left, intact PPC has been to apply inhibitory, repetitive transcranial magnetic stimulation (rTMS), which ameliorates visual neglect symptoms (see for reviews 
Cazzoli, Müri, Hess, \& Nyffeler, 2010; Hesse, Sparing, \& Fink, 2011; Müri et al., 2013; Utz, Dimova, Oppenländer, \& Kerkhoff, 2010). Importantly, the degree of behavioural amelioration correlates with the reduction in the hyperexcitability of the left, intact PPC (Koch et al., 2012). Up until now, however, it is unknown whether these positive effects of rTMS can also counteract the detrimental effects of heightened attentional load.

The aim of the present study was to investigate whether the repeated application of continuous theta burst stimulation (cTBS) - an inhibitory, patterned rTMS protocol (Huang, Edwards, Rounis, Bhatia, \& Rothwell, 2005; Nyffeler et al., 2006) - would counteract the detrimental effects of heightened attentional load on visual neglect severity. We applied cTBS because this protocol seems to be particularly promising not only in decreasing the pathological hyperexcitability of the left, intact PPC, thereby ameliorating neglect symptomatology (Cazzoli et al., 2012; Fu et al., 2015; Hopfner et al., 2014; Koch et al., 2012; Nyffeler, Cazzoli, Hess, \& Müri, 2009), but also in "stabilising and locking" the excitability of the stimulated area (Goldsworthy, Müller-Dahlhaus, Ridding, \& Ziemann, 2014a). This latter aspect has the potential to prevent the exacerbation of neglect severity, which is associated with heightened visual attentional load.

We applied cTBS over the left, contralesional PPC in a group of neglect patients, under high and low visual attentional load conditions. We measured the effects of the intervention and its interaction with the varying visual attentional load by means of two approaches. First, we administered a computerised, on-screen search task, in which visual attentional load was directly manipulated and behavioural performance was assessed. Eye 
movements were measured during the task to assess whether the overt spatial allocation of attention (i.e., the distribution of visual fixations in space) was associated with the accuracy of visual target detection (cf. Karnath, Niemeier, \& Dichgans, 1998; Malhotra, Coulthard, \& Husain, 2006; Müri, Cazzoli, Nyffeler, \& Pflugshaupt, 2009; Pflugshaupt et al., 2004; Sprenger, Kömpf, \& Heide, 2002). Second, in an additional control experiment, we administered two paper-pencil cancellation tasks that are known to impose different levels of visual attentional load, in order to test whether the ameliorative effects of cTBS were generalizable to overt visual search tasks that have a greater integrative somatomotor component, and which are often administered to assess the presence and severity of neglect. 


\section{Materials and Methods}

\subsection{Patients}

Thirteen patients with left-sided, hemispatial neglect after a subacute righthemispheric stroke were recruited. Ten out of the thirteen patients participated in only one condition of the experiment (i.e., with cTBS or with sham stimulation), whereas three patients participated in both of these conditions (i.e., cTBS and sham conditions).

Of the ten patients who participated in only one condition, five patients were randomly allocated to the cTBS condition, whereas the other five patients were allocated to the sham stimulation condition. There was no significant difference in age (52.60 years, standard error of the mean [SEM] $=5.58$; and 53.00 years, SEM $=3.65$ ) or latency between stroke onset and testing (17.40 days, $\mathrm{SEM}=2.99$; and 16.40 days, $\mathrm{SEM}=1.66)$ between these two groups $\left(\mathrm{t}_{8}=-.06\right.$, $p=.95 ;$ and $t_{8}=.29, p=.78 ; 2$-tailed).

For the three patients participating in both conditions, mean age was 54.20 years $(\mathrm{SEM}=5.44)$ and latency between stroke onset and testing was 19.00 days (SEM=3.51). Age and latency between stroke onset and testing of these three patients were not significantly different from the five patients allocated to the cTBS condition $(U=7.50, z<.001, p>.999$; and $U=7.50, z<.001, p>.999$; exact, 2-tailed) and the five patients allocated to the sham stimulation condition $(U=6.50, z=-.30, p=.875$; and $U=4.00, z=-1.04, p=.393$; exact, 2-tailed). The three patients were first tested in the sham condition (first testing session) and then, within five days, in the cTBS condition (second testing session). Their baseline performance on the Computerised Balloons test - the main outcome 
measure of the present study - was not significantly different in the first testing session from the five patients allocated to the sham stimulation condition (low/high load, and left/right side; all $U$ 's $\geq 2.00$, all $z$ 's $\geq-1.68$, all $p$ 's $\geq .143$; exact, 2-tailed), and not significantly different in the second testing session from the five patients allocated to the cTBS condition (low/high load, and left/right side; all $U$ 's $\geq 5.50$, all $z$ 's $\geq-.640$, all $p$ 's $\geq .625$; exact, 2-tailed). Moreover, the effects of sham stimulation and cTBS on performance (in terms of "high-low load difference', see Methods and Results section) were not significantly different in the three patients as compared to the two groups comprised of five patients (sham pre: $U=2.00, z=-1.724, p=.125$; sham post: $U=7.00, z=-.155, p=.893$; cTBS pre: $U=5.00, z=-.789, p=.679$; cTBS post: $U=4.50, z=-1.033, p=.464$; exact, 2-tailed).

On the basis that the three patients who participated in both conditions were comparable in all these respects (age, latency between stroke onset and testing, baseline performance, effects of brain stimulation) with the two groups comprised of five patients, we elected to pool the data for all further analyses. Neglect diagnosis was based on clinical assessment and three paper-pencil neuropsychological tests (a cancellation test, a line bisection test, and a drawing test). All patients presented deficits in at least two out of these three tests, and all patients presented deficits in the cancellation test. Results of paper-pencil clinical testing of the patients at admission are summarized in Supplementary Table 1.

All patients had normal or corrected-to-normal visual acuity. The central $30^{\circ}$ of their visual field was intact, as assessed by perimetry (Octopus or Goldman Kinetic Perimetry). 
In order to map cerebral lesion location and to calculate lesion volume, we performed lesion mapping and overlap analyses by means of the MRIcron software (Rorden et al., 2007) on high-resolution, structural MRI images that were acquired for all patients. A collaborator who was naïve with respect to both the patients' symptoms and results from the neuropsychological tests carried out lesion mapping and overlap analyses. The procedure was the same as applied by Karnath et al. (2002); namely, if a MRI sequence was conducted within the first 48 hours post-stroke, then diffusion-weighted scans were used as the basis for lesion analyses; otherwise, T2-weighted scans were used. The lesion margins were directly demarcated on every transverse slice of the individual MRI images. Then, both scans and demarcated lesions were mapped into approximate Talairach space by means of the spatial normalization algorithm integrated in SPM 5 (source: http://www.fil.ion.ucl.ac.uk/spm/). Maps displaying the overlap of patient's individual lesion locations are depicted in Fig. 1. For patients in the cTBS condition, the mean lesion volume was $59.59 \mathrm{~cm}^{3}$ $\left(\mathrm{SEM}=10.79 \mathrm{~cm}^{3}\right)$, whereas, for patients in the sham stimulation condition, the mean lesion volume was $64.78 \mathrm{~cm}^{3}\left(\mathrm{SEM}=20.59 \mathrm{~cm}^{3}\right)$. The mean lesion volume was not significantly different between the patients participating in these two conditions (i.e., cTBS, sham; $\mathrm{t}_{14}=-.223, p=.827$; 2 -tailed).

Fig. 1

Insert about here 
All patients were assessed by means of internationally accepted safety guidelines for the application of TMS application (Rossi et al., 2009), which included screening for a history of epilepsy, prior head trauma, drug and alcohol abuse, and major psychiatric disorders.

All of the patients gave written, informed consent. The study was conducted in compliance with the Declaration of Helsinki and was approved by the Ethical Committee of the State of Bern.

\subsection{Behavioural Tasks}

\subsubsection{Computerised Balloons test with eye movement recording}

An adapted, computerised version of The Balloons Test (Edgeworth, Robertson, \& McMillan, 1998) was administered. Patients were instructed to search an array of stylised balloons on a computer screen (circles with adjacent vertical lines, representing the string), in order to locate a single balloon that was not connected to a string (i.e., a simple circle). This balloon was designated as the target, whereas the other balloons with strings represented distracters. In the low load condition, the target was embedded amongst 50 distracters, whereas, in the high load condition, it was embedded amongst 100 distracters (Fig. 2). The computerised Balloons test consisted of 12 trials in the low and 12 trials in the high load condition. On each trial, the target was placed in a different location within the distracter array (determined according to an imaginary $4 \times 3$ grid; 6 targets per side).

Patients responded by pointing to the target with their right index finger. Each array remained on the screen until the patient found the target, or, until the patient declared that he/she could not locate it. To avoid fatigue, patients were 
given the option to take breaks between trials, as needed. Patients were given four practice trials ( 2 low and 2 high load).

Fig. 2

Insert about here

\subsubsection{Paper-pencil cancellation tasks}

We also administered two widely used, standardised paper-pencil tests. The low load condition was based on the Star Cancellation Test (Wilson, Cockburn, \& Halligan, 1987); the high load condition on the Random Shape Cancellation Task (Weintraub \& Mesulam, 1988). These two paper-pencil tests involved the presentation of a very similar number of targets (54 vs. 60 ; distributed equally across left and right sides of the search array), but were based on the presentation of a very different number of distracters ( 75 vs. 310 , respectively). As a consequence, the Random Shape Cancellation test has been shown to elicit more severe neglect than the Star Cancellation Test (Sarri et al., 2009). Patients were instructed to locate and cross out all of the targets on the sheet in each load condition.

\subsection{Apparatus}

Eye movements were recorded during the Balloons test, using a remote, infrared-based eye tracking system (T120, Tobii Technology). Visual stimuli 
used in the Balloons test were presented on an integrated screen (17" TFT monitor).

cTBS and sham stimulation were applied using a MagPro X100 stimulator, connected to either a round coil or a sham coil (MC-125 or MC-P-B70; Medtronic Functional Diagnostics).

\subsection{Pre-stimulation and brain stimulation experimental procedures}

The cTBS protocol consisted of 801 pulses delivered in a continuous train. The train was comprised of 267 bursts, which each contained three single pulses at $30 \mathrm{~Hz}$, repeated at $6 \mathrm{~Hz}$, and had a total duration of 44s (Cazzoli et al., 2012; Nyffeler et al., 2009). cTBS and sham were delivered over parietal electrode site P3 (International 10-20 EEG System), which overlies the left PPC in proximity of the intraparietal sulcus (Hilgetag, Théoret, \& Pascual-Leone, 2001). rTMS over this site has been reliably shown to improve hemispatial neglect (Cazzoli et al., 2012; Kim, Chun, Kim, \& Lee, 2013; Kim, Kim, Shin, \& Kim, 2010; Koch et al., 2008; Lim, Kang, \& Paik, 2010; Nyffeler et al., 2009; Song et al., 2009).

The coil was held tangentially to the scalp, with the handle pointing backwards, and the current flowing in a clockwise direction as viewed from above. The patients were asked to keep their eyes closed for the duration of the cTBS. cTBS was applied at $100 \%$ of patients' individual resting motor threshold of the right small hand muscles. Sham stimulation was applied according to the same protocol, with the exception that a sham coil was connected to the output from the stimulator. 
Respective baseline performance measures on the computerised and paperpencil behavioural tasks were acquired during a pre-stimulation phase. cTBS was administered on completion of this phase, and involved the application of two trains separated by a 15 minutes interval. This protocol significantly ameliorates the detection of left-sided targets in hemispatial neglect for up to eight hours (Nyffeler et al., 2009). Both the computerised behavioural task with eye movement measurement and the paper-pencil tasks were administered in a time window that fell well within an eight hours period, and were thus both appropriately tested against the efficacy of cTBS. The computerised and paperpencil tasks were repeated immediately after the application of cTBS in order to assess the effects on the behavioural and oculomotor performance-based measures.

\subsection{Data analyses}

The number of targets that were correctly detected on the computerised Balloons test and the mean reaction time to these targets were computed for both sides of the screen for patients in each of the two stimulation conditions, in the two load conditions, and at the pre- and post-stimulation time points. An equivalent dependent variable based on detected targets was calculated for both the Star Cancellation Test and the Random Shape Cancellation Task, as a function of stimulation condition, side, and time point. To enable a direct comparison between the paper and pencil tests, the numbers of detected targets were converted into percentages of their respective maxima.

Eye movements were recorded on the Balloons test to assess the deployment of visual attention in space. Fixations outside the screen were filtered out. 
Moreover, fixations shorter than 100 ms were excluded from the analysis (Carpenter, 1988). Percentages of visual fixations on the left and the right sides of the screen were calculated on each trial for each patient. A mean value of these percentages was then calculated for each patient as a function of each stimulation condition, in the two load conditions at the pre- and post-stimulation time points.

Separate mixed-model, repeated-measures analyses of variance (ANOVAs) were performed on the above-mentioned dependent measures, with stimulation condition (cTBS, sham) as a between-subjects factor, and time point (pre, post), load (low, high), and side (left, right) as the within-subjects factors. All post-hoc tests were performed with Newman-Keuls corrected t-tests. In order to follow up the significant effects of cTBS on left-sided target detection on the computerised Balloons test and contrast them directly with the nonsignificant effects of sham stimulation, we computed a dependent measure hereafter referred to as the, 'high-low load difference', i.e.: [number of found left-sided targets in the high load condition] - [number of found left-sided targets in the low load condition]. The high-low difference was calculated for the pre and the post time points, and for the cTBS and sham stimulation conditions. The results were analysed by means of a repeated-measures ANOVA, with the within-subjects factor time point (pre, post) and the between-subjects factor stimulation condition (cTBS, sham).

Moreover, the percentage amelioration in left-sided targets detection triggered by cTBS in the low and the high load conditions was computed as follows. First, the percentage of left-sided detected targets (i.e., the number of detected targets divided by 6 [the maximum number of targets] multiplied by 100) was 
calculated for the pre and the post time points and for the high and the low load conditions. Then, the percentage of left-sided detected targets after the stimulation was subtracted from the percentage before the stimulation, for the high and the low load conditions, resulting in the percentage amelioration. The percentage amelioration in the low and the high load conditions were compared by means of a paired-samples $t$-test.

To qualitatively depict the distribution of visual fixations (pre and post stimulation, in low and high load conditions), we plotted three-dimensional heat maps for the two stimulation conditions. In the heat maps, each pixel of the screen (with coordinates $\mathrm{x}$ and $\mathrm{y}$ ) corresponds to a given density of fixations (i.e., the number of fixations per pixel, normalized with respect to the total number of fixations), and is associated with a colour value (Wilkinson \& Friendly, 2009). Larger values are represented in yellow, smaller values in black. Moreover, a smoothing filter (Eilers \& Goeman, 2004) was applied in order to group points with similar intensity values in clusters. Visual fixations were pooled within each stimulation condition, and separate heat maps were plotted for each stimulation condition, time point, and load condition. The heat maps were generated with a Matlab script (MatWorks Inc., Natick, MA). Pearson's correlations were used to test for associations between the number of detected targets in the Balloons test and the visual fixations laterality ratio values (calculated as the number of fixations on the left screen side / the number of fixations on the right screen side; a laterality ratio value was computed for every patient and stimulation condition, the pre and poststimulation time points, and the two attentional load conditions). 


\section{Results}

\subsection{Computerised Balloons test with eye movement recording}

\subsubsection{Behavioural performance: cTBS improved target detection and there was no more significant difference between low and high load conditions}

These results of the Balloons test are depicted in Fig. 3A. The main effects of load $\left(F_{1,14}=54.69, p<.001\right)$ and side $\left(F_{1,14}=73.64, p<.001\right)$ were significant, as was the interaction between load and side $\left(F_{1,14}=12.81, p=.003\right)$. Generally, patients detected more targets on the right than on the left side in both stimulation conditions, and the number of targets detected was greater in the low than in the high load condition.

Baseline performance in the cTBS and the sham stimulation conditions was equivalent. In particular, there was no significant difference in performance in the baseline measurements between the cTBS and the sham stimulation conditions, neither for high or low load, nor for the left or the right side (all p's>.05).

Of particular note are the significant two-way interaction between the stimulation condition and time point $\left(\mathrm{F}_{1,14}=8.374, p=.012\right)$, and three-way interactions between stimulation condition, time point, and side $\left(F_{1,14}=11.46, p=.005\right)$ and between stimulation condition, time point, and load $\left(F_{1,14}=14.933, p=.002\right)$, whereas the four-way interaction between stimulation condition, time point, side, and load was not significant $\left(F_{1,14}=1.56, p=.223\right)$. Post-hoc tests revealed that 
cTBS led to a significant increase in the number of left-sided targets detected in both the low and the high load conditions (vs. the pre-stimulation baseline) ( $p=.002$ and $p<.001$, respectively), whereas there was no significant difference for the corresponding contrasts in the sham stimulation condition (all p's>.05). No significant post-hoc tests were associated with the number of right-sided targets detected, as a function of the experimental factors.

In the cTBS pre-stimulation baseline, significantly fewer targets were detected on the left side in the high as compared to the low load condition $(p<.001)$, whereas target detection performance was not significantly different between high and low load conditions after cTBS ( $p>.05)$.

In relative terms, the cTBS effects were stronger in the high than in the low load condition. The percentage amelioration in left-sided target detection was significantly greater in the high load $(m=52.08 \%, S E M=9.15)$ than in the low load $(m=25.00 \%$, SEM $=5.46)$ condition $\left(t_{7}=-5.017, p=.002\right)$. Although performance of the patients in detecting left-side targets in the low load condition after cTBS application was highly effective $(m=5.25$, SEM=.366; maximum $=6)$, it was nevertheless significantly lower than the maximum $\left(\mathrm{t}_{7}=-\right.$ 2.049, $p=.040$; one-tailed). Analyses of the eye movement data (see next Section) revealed that, even though cTBS triggered an amelioration, the spatial distribution of visual fixations after its application was still not symmetrical, as it would be expected after complete neglect remission. These elements thus strongly suggest that the difference between the strengths of the cTBS effects in the high and the low load conditions are not due to a ceiling effect in the low load condition. 
In the sham stimulation condition, the performance on the left side was significantly worse in the high than in the low load condition, both before $(p<.001)$ and after $(p<.001)$ the administration of sham stimulation.

In order to follow up these effects, we calculated and analyzed a 'high-low load difference' ([number of found left-sided targets in the high load condition] [number of found left-sided targets in the low load condition] for the pre and the post time points, and for the cTBS and sham stimulation conditions. The results are reported in Fig. 3B. No significant main effect of stimulation condition was observed $\left(F_{1,14}=1.341, p=.266\right)$, but a significant main effect of time point $\left(F_{1,14}=10.239, p=.006\right)$ and - crucially - a significant interaction between stimulation condition and time point $\left(F_{1,14}=7.522, p=.012\right)$ were found. At baseline, patients both in the sham and in the cTBS conditions exhibited a detrimental effect of attentional load, as reflected in the negative values of the 'high-low load difference', and this effect was not significantly different between the two stimulation conditions $\left(\mathrm{t}_{14}=-.475, p=.642\right)$. After the application of stimulation, the patients in the cTBS condition showed a considerable reduction of the load effect - as reflected in the values of the 'high-low load difference' that approached zero - whereas the load effect showed virtually no changes for the patients in the sham stimulation condition. Indeed, after stimulation application, the detrimental effect of load was significantly lower in the cTBS than in the sham stimulation condition $\left(\mathrm{t}_{14}=2.523, p=.024\right)$. Accordingly, testing the 'high-low load difference' values within the two stimulation conditions, i.e., before and after stimulation application, yielded significant a difference in the CTBS condition ( $\left.t_{7}=-5.017, p=.002\right)$, but not in the sham stimulation condition $\left(\mathrm{t}_{7}=-.284, \mathrm{p}=.785\right)$. 
The null effects of sham stimulation demonstrate that the significant improvement in target detection observed in the cTBS stimulation condition cannot be attributed to simple practice/re-test based effects, and is thus specific to cTBS.

Fig. 3

Insert about here

The analysis of the mean reaction times revealed a significant main effect of factors load $\left(F_{1,14}=9.863, p=.007\right)$ and side $\left(F_{1,14}=35.821, p<.001\right)$. No other effects or interaction terms were significant (all p's > .05). Irrespective of stimulation condition and time point, patients showed longer reactions time for the high than the low load condition (low load: $m=16.50$ s, SEM=1.44; high load: $\mathrm{m}=22.41 \mathrm{~s}, \mathrm{SEM}=2.04$ ), and for the left than the right side (left side: $\mathrm{m}=24.46 \mathrm{~s}$, $\mathrm{SEM}=2.22$; right side: $\mathrm{m}=14.45 \mathrm{~s}, \mathrm{SEM}=.97$ ). Taken together with the search performance results reported earlier, the reaction time based analyses suggest that overt visual search in the patients became more efficient after cTBS: the same search duration led to a significantly higher number of found targets. However, the reaction time results should be interpreted with caution, because the search time was not restricted and speed was not emphasized in the task instructions. 


\subsubsection{Eye movements: Improved target detection induced by cTBS}

was associated with a re-distribution of visual fixations towards the contralesional side of space

Analysis of the percentage of visual fixations on the left and on the right side of the screen revealed a significant main effect of side $\left(F_{1,14}=109.39, p<.001\right)$, and a significant interaction between load and side $\left(\mathrm{F}_{1,14}=21.201, p<.001\right)$.

Generally, patients displayed a rightward bias in the spatial distribution of visual fixations in both stimulation conditions, which was significantly greater in the high than in the low load condition. More relevantly, there was also a significant three-way interaction between stimulation condition, time point, and side $\left(F_{1,14}=8.179, p=.013\right)$, whereas the four-way interaction between stimulation condition, time point, side, and load was not significant $\left(F_{1,14}=1.87, p=.193\right)$. Critically, post-hoc tests revealed that the application of cTBS increased the percentage of visual fixations on the left side and decreased it on the right side of the screen, both in the low $(p=.042)$ and in the high load conditions $(p=.003)$. By contrast, the absence of a significant effect of sham stimulation on visual fixations (all p's>.05) enabled us to exclude the possibility that simple practice/re-test effects account for the lateralised re-distribution of visual fixations in the cTBS stimulation condition. These results are depicted in Fig. 4. Moreover, direct tests of the effects of cTBS vs. sham stimulation on the increased proportion of visual fixations on the left side of the screen (as calculated by subtracting the percentage of visual fixations on the left screen after cTBS or sham application from the respective percentage before application) revealed that the cTBS effects were significantly stronger than the 
sham effects in the high load condition $\left(t_{14}=2.772, p=.015\right)$, and approached significance in the low load condition $\left(\mathrm{t}_{14}=2.079, p=.057\right)$.

Fig. 4

Insert about here

Moreover, to depict the qualitatively different distribution of visual fixations poststimulation, we plotted three-dimensional heat maps representing the density of fixations in each pixel of the screen (Fig. 5).

Fig. 5

Insert about here

Importantly, the relevance of the cTBS induced re-distribution of visual fixations for the efficacy of search is underscored by the significant correlations between these two variables. Significant and strong positive correlations were observed between the laterality ratio values and the number of detected targets (low load pre: $r=.883, p<.001$; low load post: $r=.699, p=.003$, high load pre: $r=.862$, 
$p<.001$; high load post: $r=.587, p=.017$; two-tailed). Hence, the cTBS induced amelioration in search efficiency - as measured by improved target detection was mediated by a re-distribution of visual fixations towards the left side of the visual array.

\subsection{Paper-pencil cancellation tasks}

The results of the Paper-pencil cancellation tasks replicated the behavioural results of the computerised Balloons test.

There were significant main effects of load $\left(F_{1,14}=30.294, p<.001\right)$ and side $\left(F_{1,14}=42.723, p<.001\right)$, and a significant interaction between load and side $\left(F_{1,14}=8.00, p=.013\right)$. Generally, the percentage of targets detected was higher on the right than on the left side, and in the low than in the high load condition, in both stimulation conditions.

In the same way as on the computerised Balloons test, baseline performance was equivalent between the cTBS and the sham stimulation conditions, as a function of high and low load conditions and left or right side of the screen (all p's>.05).

The two-way interaction between stimulation condition and time point $\left(F_{1,14}=8.636, p=.011\right)$, and three-way interactions between stimulation condition, time point, and side $\left(F_{1,14}=13.978, p=.002\right)$ and between stimulation condition, time point, and load $\left(F_{1,14}=9.594, p=.008\right)$ were significant, whereas the fourway interaction between stimulation condition, time point, side, and load was not significant $\left(\mathrm{F}_{1,14}=.065, p=.802\right)$. Post hoc tests revealed that cTBS led to a significant increase in the percentage of left-sided targets that were detected in both the low and the high load conditions (vs. the pre-stimulation baseline) 
( $p=.001, p<.001$, respectively), whereas there was no significant difference for the corresponding contrasts in the sham group (all p's>.05). No significant posthoc tests were observed for the number of detected right-sided targets.

In the cTBS pre-stimulation baseline, a significantly lower percentage of targets was detected on left side in the high as compared to the low load condition $(p<.001)$, whereas target detection performance was not significantly different between high and low load conditions after cTBS ( $p>.05)$. In the sham group, the performance on the left side was significantly worse in the high than in the low load condition, both before $(p=.001)$ and after $(p=.005)$ the administration of sham stimulation. These results are depicted in Fig. 6.

Fig. 6

Insert about here 


\section{Discussion}

In the present study, we demonstrated for the first time that the repeated cTBS applied over the left, contralesional PPC eliminated the detrimental effect of visual attentional load on neglect severity in overt visual search, whereas sham stimulation had no significant effect. Before cTBS, patients performed significantly worse in the high than in the low load condition. After cTBS, search efficiency improved significantly and target detection was equated across high and low attentional load conditions. The cTBS-induced proportional improvement in search was thus significantly greater in the high as compared to the low load condition. Importantly, visual search efficiency was significantly correlated with the redeployment of visual fixations to the contralesional visual field. The cTBS-induced load-independent behavioural amelioration was also confirmed in the additional control experiment with two different paper-pencil cancellation tasks. Importantly, these tasks are known to impose different visual attentional load levels, and are widely used to assess the presence and severity of hemispatial neglect. After cTBS, cancellation was significantly improved in both a paper-pencil cancellation test imposing a low visual attentional load (Star cancellation test) and in one imposing a high visual attentional load (Random shape cancellation task). The improvement in the paper-pencil cancellation task with high visual attentional load was proportionally greater than in the one with low visual attentional load, and the performance was no longer significantly different between these two levels of visual attentional load. This pattern closely corresponds with the results obtained in our main experiment, where visual 
attentional load was directly manipulated. The convergence across two different sets of paradigms strongly corroborates our principal findings, and shows their generalizability to overt visual search tasks that are associated with a significant somatomotor component.

It is to note that no significant cTBS effects were observed for the right-sided, ipsilesional space. This aspect is important for at least two reasons. First, the fact that the positive effects of cTBS are confined to the left side of space underlines the specificity of the findings. Second, the lack of negative effects on the right, ipsilesional side of space helps to rule out potential detrimental effects of cTBS application over the left, contralesional PPC.

The neurophysiological substrate of the stronger ameliorative effect in the high attentional load is, at present, unclear. One candidate explanation is that the repeated cTBS application over the left, intact PPC in hemispatial neglect is not only able to decrease the pathological hyperexcitability of this area (Koch et al., 2012), but also to "stabilise and lock" its excitability at a lower level. This would prevent heightened visual attentional load triggering a further increase of the excitability level in this cortical area, which may exacerbate the interhemispheric inhibitory imbalance. This line of reasoning would be in line with the recently described neurophysiological properties of repeated cTBS application in the motor system. Goldsworthy and colleagues (Goldsworthy et al., 2014a) demonstrated that when cTBS was applied twice with an interval of ten minutes, the MEP depression was resistant against the subsequent increase of physiological activity, both triggered voluntarily (i.e., by means of a voluntary muscle contraction) or externally (i.e., by means of the additional application of intermittent TBS, iTBS, an excitatory stimulation protocol). 
By extension, in the present study, the repeated application of two cTBS trains may have limited the likelihood of heightened visual attentional load inducing additional pathological hyperexcitability within the left, contralesional PPC. Additional evidence consistent with this interpretation comes from animal models used to study LTP/LTD-like phenomena associated with late-phase synaptic plasticity mechanisms (Goldsworthy, Pitcher, \& Ridding, 2014b; Huang, Chen, Rothwell, \& Wen, 2007; Huang, Rothwell, Chen, Lu, \& Chuang, 2011). For instance, in the developing visual system of the Xenopus, synaptic modifications are abolished by subsequent activity (either spontaneous or triggered by visual stimuli), unless repeated patterns of stimulation are applied (Zhou, Tao, \& Poo, 2003). Similarly, as shown in mouse hippocampal slices, repeated stimulation trains trigger a conspicuous resistance against synaptic de-potentiation (Woo \& Nguyen, 2003).

The present study also showed specific effects in the eye movement patterns, in particular, in the spatial distribution of visual fixations. The distribution of visual fixations has been often employed to study spatial biases of overt visual attention allocation in neglect, and a conspicuous rightward bias in this distribution represents a robust finding in the literature (Karnath et al., 1998; Malhotra et al., 2006; Müri et al., 2009; Pflugshaupt et al., 2004; Sprenger et al., 2002). The rightward bias in the distribution of visual fixations observed in the present study is thus in line with previous reports. However, the present study is the first to show two new findings linked to the effects of visual attentional load and to the application of cTBS in neglect on the distribution of visual fixations. First, the rightwards bias in the spatial distribution of visual fixations was modulated by visual attentional load; i.e., it was greater in the high than in the 
low load condition. Second, and more crucially, cTBS triggered a significant and specific redeployment of visual fixations towards the left, contralesional side of space, which was significantly correlated with visual search efficiency. Importantly, therefore, the redeployment of visual fixations can be seen as a candidate mechanism for the improvement in target detection on the computerbased, overt visual search task. To the best of our knowledge, the present study is the first to link a cTBS-induced amelioration of visual neglect severity with the redeployment of eye movements to the contralesional side of space. It is also important to note that in earlier studies involving neglect patients or healthy controls (e.g., Russell, Malhotra, Deidda, \& Husain, 2013; Russell, Malhotra, \& Husain, 2004; Schwartz et al., 2005; Vuilleumier et al., 2008), attentional load was manipulated centrally; i.e., gaze was kept at central fixation and attentional deployment in space operated in a covert fashion (i.e., without eye movements). In the present study, participants were directed to move their eyes to overtly deploy visuospatial attention, while attentional load was manipulated by means of the number of distracters. This approach was adopted because overt visual search occurs under naturalistic conditions (e.g., Land, 2006), and, crucially, is used to assess neglect in clinical settings (e.g., by means of cancellation tests, as applied in the present study). In future studies, it would be very interesting to assess whether cTBS also has similar ameliorative effects in visual search tasks that are based on the covert reorienting of visuospatial attention. Moreover, it would be interesting to combine cTBS with electrophysiological methods (Koch et al., 2008, 2012), resting state MRI (Carter et al., 2010), or EEG (Rizk, Ptak, Nyffeler, Schnider, \& Guggisberg, 2013) in order to investigate the relationship between cTBS, visual attentional 
load and their neurophysiologic substrate at a network level. Furthermore, cTBS effects on attentional load could be measured over a longer time period in a large patient sample, and these effects could be assessed during the activities of daily living.

In conclusion, the present study demonstrates that cTBS can be applied to reduce the detrimental effects of variable visual attentional load in hemispatial neglect on different types of overt visual search tasks. This finding may be relevant for the future rehabilitation of neglect, since variations in attentional load - which potentially trigger fluctuations in neglect severity - often occur in everyday life. 


\section{Acknowledgements}

This work was supported by the Swiss National Science Foundation (grant numbers 320030_140696; PZ00P3_154714 to D.C.).

The funding source had no involvement in study design; in the collection, analysis, and interpretation of data; in the writing of the report; or in the decision to submit the paper for publication. 


\section{References}

Baldassarre, A., Ramsey, L., Hacker, C. L., Callejas, A., Astafiev, S. V., Metcalf, N. V., Zinn, K., Rengachary, J., Snyder, A. Z., Carter, A. R., Shulman, G. L., \& Corbetta, M. (2014). Large-scale changes in network interactions as a physiological signature of spatial neglect. Brain, 137(Pt 12), 3267-3283.

Bonato, M. (2012). Neglect and extinction depend greatly on task demands: a review. Frontiers in Human Neuroscience, 6, 195.

Carpenter, R. H. S. (1988). Movements of the eyes. London (UK): Pion Ltd.

Carter, A. R., Astafiev, S. V., Lang, C. E., Connor, L. T., Rengachary, J., Strube, M. J., Pope, D. L., Shulman, G. L., \& Corbetta, M. (2010). Resting interhemispheric functional magnetic resonance imaging connectivity predicts performance after stroke. Annals of Neurology, 67(3), 365-375.

Cazzoli, D., Müri, R. M., Hess, C. W., \& Nyffeler, T. (2010). Treatment of hemispatial neglect by means of rTMS--a review. Restorative Neurology and Neuroscience, 28(4), 499-510.

Cazzoli, D., Müri, R. M., Schumacher, R., von Arx, S., Chaves, S., Gutbrod, K., Bohlhalter, S., Bauer, D., Vanbellingen, T., Bertschi, M., Kipfer, S., Rosenthal, C. R., Kennard, C., Bassetti, C. L., \& Nyffeler, T. (2012). Theta burst stimulation reduces disability during the activities of daily living in spatial neglect. Brain, 135(Pt 11), 3426-3439. 
Corbetta, M., Kincade, M. J., Lewis, C., Snyder, A. Z., \& Sapir, A. (2005).

Neural basis and recovery of spatial attention deficits in spatial neglect. Nature Neuroscience, 8(11), 1603-1610.

Corbetta, M., \& Shulman, G. L. (2011). Spatial neglect and attention networks. Annual Review of Neuroscience, 34, 569-599.

Doricchi, F., Thiebaut de Schotten, M., Tomaiuolo, F., \& Bartolomeo, P. (2008). White matter (dis)connections and gray matter (dys)functions in visual neglect: gaining insights into the brain networks of spatial awareness. Cortex, 44(8), 983-995.

Edgeworth, J., Robertson, I. H., \& McMillan, T. (1998). The Balloons Test. Bury St. Edmunds (UK): Thames Valley Test Company.

Eilers, P. H. C., \& Goeman, J. J. (2004). Enhancing scatterplots with smoothed densities. Bioinformatics, 20(5), 623-682.

Fu, W., Song, W., Zhang, Y., Yang, Y., Huo, S., Zhang, R., \& Wang, M. (2015). Long-term effects of continuous theta-burst stimulation in visuospatial neglect. The Journal of International Medical Research, 43(2), 196-203.

Goldsworthy, M. R., Müller-Dahlhaus, F., Ridding, M. C., \& Ziemann, U. (2014a). Resistant Against De-depression: LTD-Like Plasticity in the Human Motor Cortex Induced by Spaced cTBS. Cerebral Cortex. [Epub ahead of print]. 
Goldsworthy, M. R., Pitcher, J. B., \& Ridding, M. C. (2014b). Spaced Noninvasive Brain Stimulation: Prospects for Inducing Long-Lasting Human Cortical Plasticity. Neurorehabilitation and Neural Repair. [Epub ahead of print].

He, B. J., Snyder, A. Z., Vincent, J. L., Epstein, A., Shulman, G. L., \& Corbetta, M. (2007). Breakdown of functional connectivity in frontoparietal networks underlies behavioral deficits in spatial neglect. Neuron, 53(6), 905-918.

Heilman, K. M., Watson, R. T., \& Valenstein, E. (1993). Neglect and related disorders. In K. M. Heilman \& E. Valenstein (Eds.), Clinical Neuropsychology (pp. 243-294). New York (NY): Oxford University Press.

Hesse, M. D., Sparing, R., \& Fink, G. R. (2011). Ameliorating spatial neglect with non-invasive brain stimulation: from pathophysiological concepts to novel treatment strategies. Neuropsychological Rehabilitation, 21(5), 676-702.

Hilgetag, C. C., Théoret, H., \& Pascual-Leone, A. (2001). Enhanced visual spatial attention ipsilateral to rTMS-induced "virtual lesions" of human parietal cortex. Nature Neuroscience, 4(9), 953-957.

Hopfner, S., Cazzoli, D., Müri, R. M., Nef, T., Mosimann, U. P., Bohlhalter, S., Vanbellingen, T., \& Nyffeler, T. (2014). Enhancing treatment effects by combining continuous theta burst stimulation with smooth pursuit training. Neuropsychologia. [Epub ahead of print]. 
Huang, Y.-Z., Chen, R.-S., Rothwell, J. C., \& Wen, H.-Y. (2007). The after-effect of human theta burst stimulation is NMDA receptor dependent. Clinical Neurophysiology, 118(5), 1028-1032.

Huang, Y.-Z., Edwards, M. J., Rounis, E., Bhatia, K. P., \& Rothwell, J. C. (2005). Theta burst stimulation of the human motor cortex. Neuron, 45(2), 201206.

Huang, Y.-Z., Rothwell, J. C., Chen, R.-S., Lu, C.-S., \& Chuang, W.-L. (2011). The theoretical model of theta burst form of repetitive transcranial magnetic stimulation. Clinical Neurophysiology, 122(5), 1011-1018.

Husain, M., \& Kennard, C. (1996). Visual neglect associated with frontal lobe infarction. Journal of Neurology, 243(9), 652-657.

Jovicich, J., Peters, R. J., Koch, C., Braun, J., Chang, L., \& Ernst, T. (2001). Brain areas specific for attentional load in a motion-tracking task. Journal of Cognitive Neuroscience, 13(8), 1048-1058.

Karnath, H.-O., Fruhmann Berger, M., Küker, W., \& Rorden, C. (2004). The anatomy of spatial neglect based on voxelwise statistical analysis: a study of 140 patients. Cerebral Cortex, 14(10), 1164-1172. 
Karnath, H.-O., Himmelbach, M., \& Rorden, C. (2002). The subcortical anatomy of human spatial neglect: putamen, caudate nucleus and pulvinar. Brain, 125(Pt 2), 350-360.

Karnath, H.-O., Niemeier, M., \& Dichgans, J. (1998). Space exploration in neglect. Brain, 121 (Pt 12), 2357-2367.

Karnath, H.-O., Rorden, C., \& Ticini, L. F. (2009). Damage to white matter fiber tracts in acute spatial neglect. Cerebral Cortex, 19(10), 2331-2337.

Kim, B. R., Chun, M. H., Kim, D.-Y., \& Lee, S. J. (2013). Effect of high- and lowfrequency repetitive transcranial magnetic stimulation on visuospatial neglect in patients with acute stroke: a double-blind, sham-controlled trial. Archives of Physical Medicine and Rehabilitation, 94(5), 803-807.

Kim, J. S., Kim, J. C., Shin, S. H., \& Kim, Y. K. (2010). Comparison of Effects of Repetitive Transcranial Magnetic Stimulation with High- or Low-frequency on Visuospatial Neglect in Stroke Patients. Journal of Korean Academy of Rehabilitation Medicine, 34(4), 397-402.

Koch, G., Bonnì, S., Giacobbe, V., Bucchi, G., Basile, B., Lupo, F., Versace, V., Bozzali, M., \& Caltagirone, C. (2012). $\theta$-burst stimulation of the left hemisphere accelerates recovery of hemispatial neglect. Neurology, 78(1), 24-30. 
Koch, G., Cercignani, M., Bonnì, S., Giacobbe, V., Bucchi, G., Versace, V., Caltagirone, C., \& Bozzali, M. (2011). Asymmetry of parietal interhemispheric connections in humans. The Journal of Neuroscience, 31(24), 8967-8975.

Koch, G., Oliveri, M., Cheeran, B., Ruge, D., Lo Gerfo, E., Salerno, S., Torriero, S., Marconi, B., Mori, F., Driver, J., Rothwell, J. C., \& Caltagirone, C. (2008). Hyperexcitability of parietal-motor functional connections in the intact lefthemisphere of patients with neglect. Brain, 131(Pt 12), 3147-3155.

Land, M. F. (2006). Eye movements and the control of actions in everyday life. Progress in Retinal and Eye Research, 25(3), 296-324.

Lim, J. Y., Kang, E. K., \& Paik, N.-J. (2010). Repetitive transcranial magnetic stimulation to hemispatial neglect in patients after stroke: an open-label pilot study. Journal of Rehabilitation Medicine, 42(5), 447-452.

Malhotra, P., Coulthard, E., \& Husain, M. (2006). Hemispatial neglect, balance and eye-movement control. Current Opinion in Neurology, 19(1), 14-20.

Mort, D. J., Malhotra, P., Mannan, S. K., Rorden, C., Pambakian, A., Kennard, C., \& Husain, M. (2003). The anatomy of visual neglect. Brain, 126(Pt 9), 19861997. 
Müri, R. M., Cazzoli, D., Nef, T., Mosimann, U. P., Hopfner, S., \& Nyffeler, T. (2013). Non-invasive brain stimulation in neglect rehabilitation: an update. Frontiers in Human Neuroscience, 7, 248.

Müri, R. M., Cazzoli, D., Nyffeler, T., \& Pflugshaupt, T. (2009). Visual exploration pattern in hemineglect. Psychological Research, 73(2), 147-157.

Nyffeler, T., Cazzoli, D., Hess, C. W., \& Müri, R. M. (2009). One session of repeated parietal theta burst stimulation trains induces long-lasting improvement of visual neglect. Stroke, 40(8), 2791-2796.

Nyffeler, T., Wurtz, P., Lüscher, H.-R., Hess, C. W., Senn, W., Pflugshaupt, T., von Wartburg, R., Lüthi, M., \& Müri, R. M. (2006). Extending lifetime of plastic changes in the human brain. The European Journal of Neuroscience, 24(10), 2961-2966.

Pflugshaupt, T., Bopp, S. A., Heinemann, D., Mosimann, U. P., von Wartburg, R., Nyffeler, T., Hess, C. W., \& Müri, R. M. (2004). Residual oculomotor and exploratory deficits in patients with recovered hemineglect. Neuropsychologia, 42(9), 1203-1211.

Rizk, S., Ptak, R., Nyffeler, T., Schnider, A., \& Guggisberg, A. G. (2013). Network mechanisms of responsiveness to continuous theta-burst stimulation. The European Journal of Neuroscience, 38(8), 3230-3238. 
Rossi, S., Hallett, M., Rossini, P. M, Pascual-Leone, A., \& Safety of TMS Consensus Group (2009). Safety, ethical considerations, and application guidelines for the use of transcranial magnetic stimulation in clinical practice and research. Clinical Neurophysiology, 120(12), 2008-2039.

Russell, C., Malhotra, P., Deidda, C., \& Husain, M. (2013). Dynamic attentional modulation of vision across space and time after right hemisphere stroke and in ageing. Cortex, 49(7), 1874-1883.

Russell, C., Malhotra, P., \& Husain, M. (2004). Attention modulates the visual field in healthy observers and parietal patients. Neuroreport, 15(14), 2189_ 2193.

Sarri, M., Greenwood, R., Kalra, L., \& Driver, J. (2009). Task-related modulation of visual neglect in cancellation tasks. Neuropsychologia, 47(1), 91-103.

Schwartz, S., Vuilleumier, P., Hutton, C., Maravita, A., Dolan, R. J., \& Driver, J. (2005). Attentional load and sensory competition in human vision: modulation of fMRI responses by load at fixation during task-irrelevant stimulation in the peripheral visual field. Cerebral Cortex, 15(6), 770-786.

Shinoura, N., Suzuki, Y., Yamada, R., Tabei, Y., Saito, K., \& Yagi, K. (2009). Damage to the right superior longitudinal fasciculus in the inferior parietal lobe plays a role in spatial neglect. Neuropsychologia, 47(12), 2600-2603. 
Song, W., Du, B., Xu, Q., Hu, J., Wang, M., \& Luo, Y. (2009). Low-frequency transcranial magnetic stimulation for visual spatial neglect: a pilot study. Journal of Rehabilitation Medicine, 41(3), 162-165.

Sprenger, A., Kömpf, D., \& Heide, W. (2002). Visual search in patients with left visual hemineglect. Progress in Brain Research, 140, 395-416.

Utz, K. S., Dimova, V., Oppenländer, K., \& Kerkhoff, G. (2010). Electrified minds: transcranial direct current stimulation (tDCS) and galvanic vestibular stimulation (GVS) as methods of non-invasive brain stimulation in neuropsychology--a review of current data and future implications. Neuropsychologia, 48(10), 2789-2810.

Vuilleumier, P., Schwartz, S., Verdon, V., Maravita, A., Hutton, C., Husain, M., \& Driver, J. (2008). Attention-dependent functional abnormality in retinotopic visual cortex for patients with parietal lesions and spatial neglect. Current Biology, 18(19), 1525-1529.

Weintraub, S., \& Mesulam, M. M. (1988). Visual hemispatial inattention: stimulus parameters and exploratory strategies. Journal of Neurology, Neurosurgery, and Psychiatry, 51(12), 1481-1488.

Wilkinson, L., \& Friendly, M. (2009). The History of the Cluster Heat Map. American Statistician, 63(2), 179-184. 
Wilson, B., Cockburn, J., \& Halligan, P. W. (1987). Behavioural inattention test. Titchfield (UK): Thames Valley Test Company.

Woo, N. H., \& Nguyen, P. V. (2003). Protein synthesis is required for synaptic immunity to depotentiation. The Journal of Neuroscience, 23(4), 1125-1132.

Zhou, Q., Tao, H. W., \& Poo, M. (2003). Reversal and stabilization of synaptic modifications in a developing visual system. Science, 300(5627), 1953-1957. 


\section{Figure captions}

Fig. 1.

Overlap maps displaying the lesion location for patients in the cTBS condition (upper row) and in the sham stimulation condition (lower row). The lesion overlap is plotted onto the ch2.nii.gz template of the MNI standard brain, using axial slices oriented according to the neurological convention. The slices are depicted in $8 \mathrm{~mm}$ ascending steps, and the z-position in the MNI Talairach stereotaxic space is presented at the bottom of the figures. The number of patients with damage to a specific region is colour-coded according to the legend. Note: five patients participated only in the cTBS or the sham condition, respectively, whereas three patients participated in both conditions.

Fig. 2.

Two example search arrays used on the computer-based visual search task. Low load condition (left panel; 50 distracters; 1 target) and high load condition (right panel; 100 distracters; 1 target). The task was an adapted and computerised version of The Balloons Test (Edgeworth et al., 1998). Patients were asked to find a single balloon in the array that did not have a string, and was designated as the target (located on the left, upper quadrant of these two example arrays). Eye movements were recorded during the test in order to assess the spatial deployment of visual attention. 


\section{Fig. 3.}

Target detection performance in the Balloons test (computer-based visual search task). A. Mean number of targets detected in the Balloons test. Results are shown for the cTBS condition (upper panels) and for the sham stimulation condition (lower panels); before and after intervention ('pre' and 'post' cTBS or sham stimulation); for the low load and the high load conditions (left and right panel, respectively); and for the left and the right side. B. Results of the analysis of the 'high-low load difference' (calculated as: [number of found left-sided targets in the high load condition] - [number of found left-sided targets in the low load condition]), for the pre and the post time points, and for the cTBS and sham stimulation conditions. Error bars depict the standard error of the mean (SEM). Asterisks denote significant post-hoc tests $\left({ }^{* *} p<.01\right.$; $\left.{ }^{*} p<.05\right)$; ns: not significant.

Fig. 4.

Mean percentage of visual fixations on the left and the right side of the screen. Results are shown as a function of cTBS and sham stimulation conditions, before and after intervention ('pre' and 'post' stimulation), and for the low load condition (left panels) and the high load condition (right panels). Error bars depict the standard error of the mean (SEM). Asterisks denote significant post-hoc tests $\left({ }^{* *} p<.01 ;{ }^{*} p<.05\right)$.

Fig. 5.

Spatial distributions of visual fixations on the horizontal and vertical dimensions during the computer-based visual search task, represented by 
means of heat maps. Heat maps are plotted for cTBS (upper panels) and sham (lower panels) stimulation conditions, as a function of time point ('pre' and 'post' stimulation) and load condition (low load and high load conditions). Each pixel of the screen has a given density of fixations (normalized with respect to the total number of fixations) and is associated with a colour value. Larger values are represented in yellow, smaller values in black.

In the cTBS condition, the graphs of the baseline distributions ('pre') show a considerable accumulation of visual fixation densities towards the right side of the screen, which is particularly evident in the high load condition. After cTBS application ('post'), the visual fixations densities are more evenly distributed across both sides of the screen. Before stimulation, the areas with low visual fixation densities (i.e., less than 10\%, represented in black on the heat maps) which are located most predominantly on the left screen half - occupied $17.27 \%$ of the screen in the low load condition and $34.36 \%$ of the screen in the high load condition. After cTBS application, these areas were reduced in extent, with values of $10.42 \%$ of the screen in the low load condition and $17.85 \%$ in the high load condition.

In the sham stimulation condition, the graphs of the baseline distributions ('pre' stimulation) show a very similar pattern as seen in the cTBS condition. However, after the application of sham stimulation, the changes in these distributions are much smaller than in the cTBS condition. Indeed, the areas with low visual fixation densities represented $14.52 \%$ of the screen in the low load condition and $25.10 \%$ of the screen in the high load condition before stimulation. After sham stimulation, these areas remained quite similar in extent 
relative to the baseline distributions, with values of $15.03 \%$ of the screen in the low load condition and $20.00 \%$ in the high load condition.

Fig. 6.

Mean percentage of targets that were detected in the paper-pencil cancellation tasks. Low load: Star Cancellation Test; high load: Random Shape Cancellation Task. The numbers of detected targets were converted into percentages of their respective maxima to allow a direct comparison between the two tests. Results are shown for the cTBS condition (upper panels) and for the sham stimulation condition (lower panels); before and after intervention ('pre' and 'post' cTBS or sham stimulation); for the low load and the high load conditions (left and right panel, respectively); and for the left and the right side. Error bars depict the standard error of the mean (SEM). Asterisks denote significant post-hoc tests $\left({ }^{* *} p<.01\right)$; ns: not significant. 


\section{Abbreviations}

$\begin{array}{ll}\text { ANOVA } & \text { Analysis of variance } \\ \text { cTBS } & \text { Continuous theta burst stimulation } \\ \text { iTBS } & \text { Intermittent theta burst stimulation } \\ \text { PPC } & \text { Posterior parietal cortex } \\ \text { rTMS } & \text { Repetitive transcranial magnetic stimulation } \\ \text { SEM } & \text { Standard error of the mean }\end{array}$


Supplementary Table 1. Results of paper-pencil clinical testing of the patients at admission ${ }^{1}$.

\begin{tabular}{|c|c|c|c|c|c|c|c|}
\hline \multirow{2}{*}{ Pat. No. } & \multirow{2}{*}{ Sex } & \multicolumn{2}{|c|}{ Cancellation test } & \multicolumn{2}{|c|}{ Line bisection test } & \multicolumn{2}{|c|}{ Drawing test } \\
\hline & & Type $^{2}$ & $\mathrm{CoC}^{3}$ & Type $^{4}$ & $\begin{array}{c}\text { Mean \% } \\
\text { deviation }\end{array}$ & Type $^{6}$ & Score $^{7}$ \\
\hline 1 & $m$ & $\mathrm{BII}$ & 0.709 & $\mathrm{Cmpl}$ & 7.39 & CplSc & $1 \mathrm{~L}$ \\
\hline 2 & $\mathrm{~m}$ & RSC & 0.221 & $\mathrm{Cmpl}$ & 4.48 & CER; 5-pt & $2 \mathrm{~L} ; 2 \mathrm{R}$ \\
\hline 3 & $\mathrm{~m}$ & RSC & 0.925 & $\mathrm{Cmpl}$ & 56.84 & CER & $2 \mathrm{~L}$ \\
\hline 4 & w & RSC & 0.264 & $\mathrm{Cmpl}$ & 12.98 & CER & $2 \mathrm{~L}$ \\
\hline 5 & $\mathrm{~m}$ & BII & 0.093 & $\mathrm{Cmpl}$ & 32.44 & $\mathrm{SCD} ; \mathrm{Clk}$ & $2 \mathrm{~L} ; 2 \mathrm{~L}$ \\
\hline 6 & w & RSC & 0.286 & $\mathrm{Cmpl}$ & 11.16 & CER & 0 \\
\hline 7 & $\mathrm{~m}$ & RSC & 0.577 & $\mathrm{Cmpl}$ & 10.42 & 5-pt & $2 \mathrm{R}$ \\
\hline 8 & $\mathrm{~m}$ & RSC & 0.245 & $\mathrm{Cmpl}$ & 9.45 & SCD; 5-pt & $2 \mathrm{~L} ; 2 \mathrm{R}$ \\
\hline 9 & w & StrC & 0.304 & Schnk & 10.84 & CER & $1 \mathrm{~L}$ \\
\hline 10 & $\mathrm{~m}$ & RSC & 0.149 & $\mathrm{Cmpl}$ & 14.92 & CER & 0 \\
\hline 11 & $\mathrm{~m}$ & $\mathrm{BII}$ & 0.393 & $\mathrm{Cmpl}$ & 4.81 & SCD; 5-pt & $1 \mathrm{~L} ; 2 \mathrm{R}$ \\
\hline 12 & $\mathrm{~m}$ & $\mathrm{BII}$ & 0.144 & $\mathrm{Cmpl}$ & 9.72 & SCD; 5-pt & $1 \mathrm{~L} ; 1 \mathrm{R}$ \\
\hline 13 & $\mathrm{~m}$ & $\mathrm{BII}$ & 0.101 & Schnk & 10.67 & Rey & $2 \mathrm{~L}$ \\
\hline
\end{tabular}

\section{Notes:}

${ }^{1}$ The mean time between admission and experimental measures was 5.75 days (SEM $=0.642$ ), and no significant difference between the cTBS and the sham stimulation conditions was observed concerning this parameter $\left(\mathrm{t}_{14}=.768, p=.455\right)$.

2 BII = Bells Test, according to: Gauthier L, Dehaut F, Joanette $Y$. The bells test: A quantitative and qualitative test for visual neglect. Int J Clin Neuropsychol 1989; 11: 49-54. RSC = Random Shape Cancellation Test, according to: Weintraub S, Mesulam MM. Visual hemispatial inattention: Stimulus parameters and exploratory strategies. J Neurol Neurosurg Psychiatry 1988; 51: 1481-8. StrC = Star cancellation subtest from the Behavioural Inattention Test (BIT), according to: Wilson B, Cockburn J, Halligan PW. Behavioural Inattention Test. Titchfield, UK: Thames Valley Test Company; 1987.

${ }^{3} \mathrm{CoC}=$ Center of Cancellation index. Cut-off set at 0.0809 . According to: Rorden C, Karnath HO. A simple measure of neglect severity. Neuropsychologia 2010; 48: 2758-63. No significant difference between the CTBS and the sham stimulation conditions was observed concerning the CoC in the cancellation test $\left(\mathrm{t}_{14}=.066, p=.948\right)$.

${ }^{4} \mathrm{Cmpl}=$ Complex line bisection test, according to: Butter CM, Mark VW, Heilman KM. An experimental analysis of factors underlying neglect in line bisection. J Neurol Neurosurg Psychiatry 1988; 51: 1581-3. Schnk = Line bisection test, according to: Schenkenberg T, Bradford DC, Ajax ET. Line bisection and unilateral visual neglect in patients with neurologic impairment. Neurology 1980; 30: 509-17.

5 Mean \% deviation in the line bisection test. Cut-off deviation set at $11 \%$ (according to: Schenkenberg T, Bradford DC, Ajax ET. Line bisection and unilateral visual neglect in patients with neurologic impairment. Neurology 1980; 30: 509-17). No significant difference between the cTBS and the sham stimulation conditions was observed concerning the mean $\%$ deviation in the line bisection test $\left(\mathrm{t}_{14}=-1.252, p=.231\right)$.

${ }^{6}$ CER = Figure Copying test of the CERAD, according to: Morris JC, Heyman A, Mohs RC, Hughes JP, van Belle G, Fillenbaum G, Mellits ED, Clark C, CERAD investigators. The Consortium to Establish a Registry for Alzheimer's Disease (CERAD). Part 1. Clinical and neuropsychological assessment of Alzheimer's disease. Neurology 1989; 39: 1159-65. Clk = Clock drawing task. CpISc = Copy of a complex scene (house, threes, fence), according to: Gainotti G, Messerli P, Tissot R. Qualitative analysis of unilateral spatial neglect in relation to laterality of cerebral lesions. $J$ Neurol Neurosurg Psychiatry 1972; 35: 545-50. Rey = Rey-Osterrieth Complex Figure Test, copy, according to: Osterrieth PA. Le test de copie d'une figure complexe. Arch Psychol 1944; 30: 206356. SCD = Copy drawing task (star, cube, daisy), according to: Halligan PW, Cockburn J, Wilson BA. The behavioural assessment of visual neglect. Neuropsychol Rehabil 1991; 1: 5-32. 5-pt = 
Five-point Test, according to: Regard M, Strauss E, Knapp P. Children's production on verbal and non-verbal fluency tasks. Percept Mot Skills 1982; 55: 839-44.

${ }^{7}$ For CER, Clk, CplSc, Rey, and SCD performance was scored as: $0=$ intact; $1 \mathrm{~L}=$ distorted left side; $2 \mathrm{~L}=$ flagrant omission(s) on the left side. For 5 -pt performance was scored as: $0=$ symmetric production (balanced number of drawings on the left and right side of the sheet); $1 \mathrm{R}=$ moderately asymmetric production towards the right (more drawing on the right side of the sheet, some squares are left blank on the left side of the sheet); $2 \mathrm{R}$ = substantially asymmetric production (drawings are produced almost exclusively or exclusively on the right side of the sheet). No significant difference between the CTBS and the sham stimulation conditions was observed in terms of the presence of an impairment in the drawing test $\left(\chi^{2}(1)=.410, p=.522\right)$. 
Figure 1

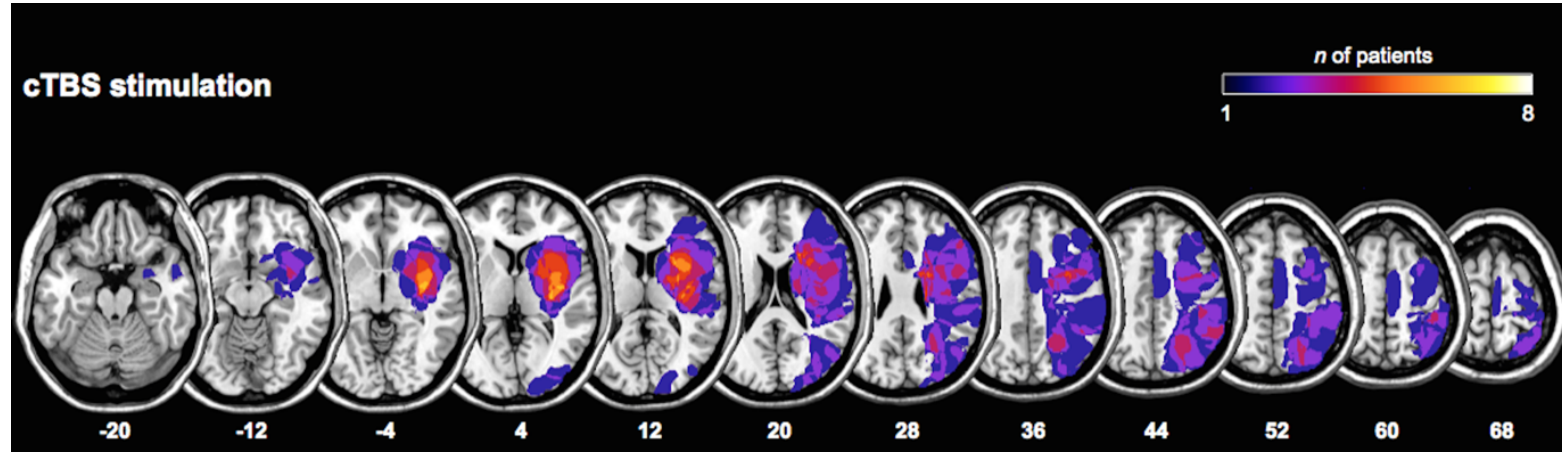

Sham stimulation
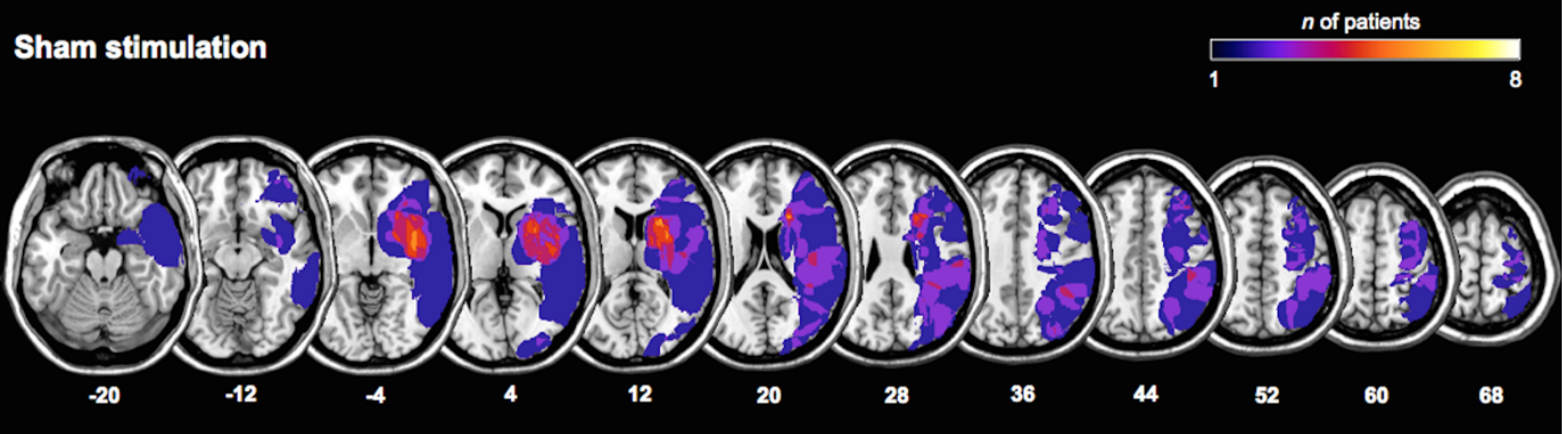
Figure 2

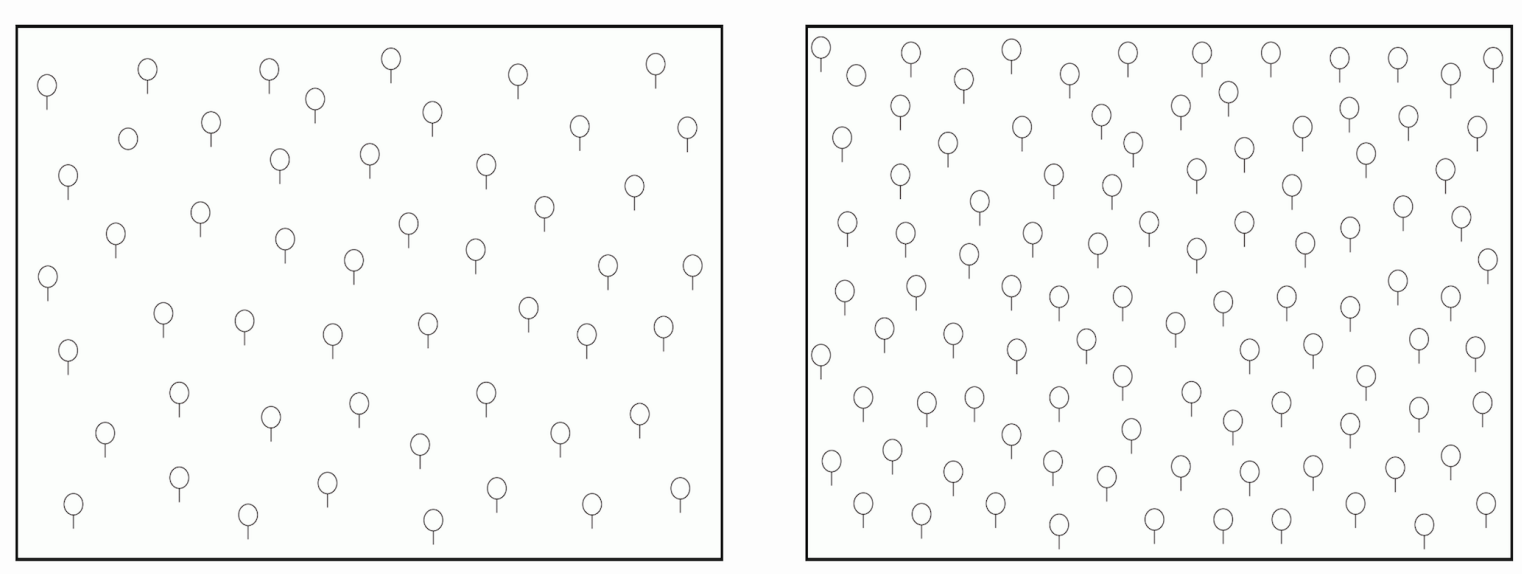


Figure 3

\section{Balloons test}

A

Low load

High load

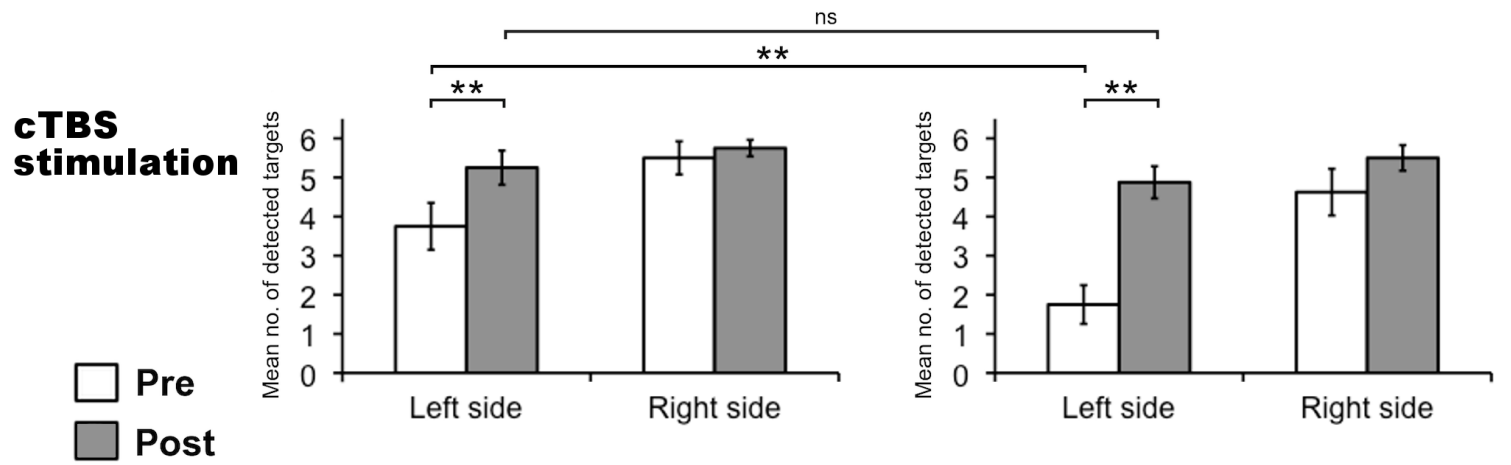

Post

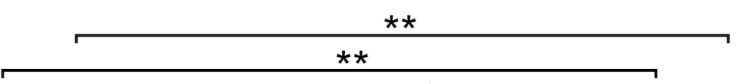

sham

stimulation
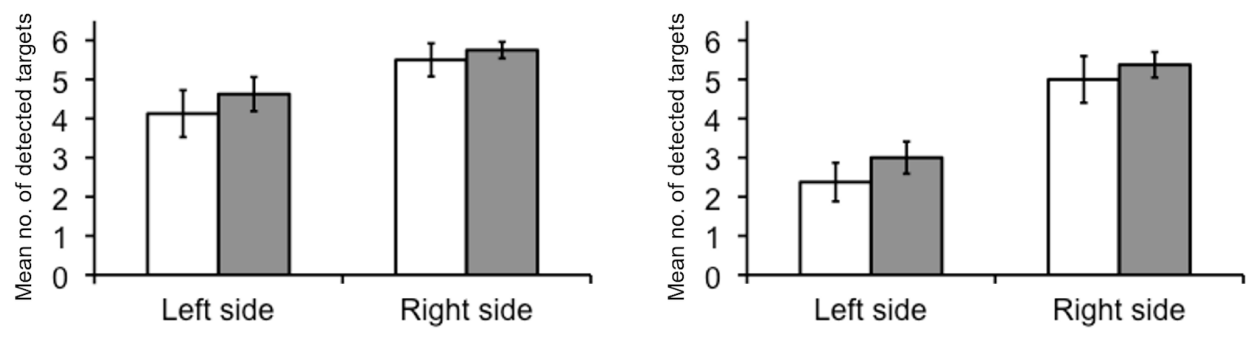

B

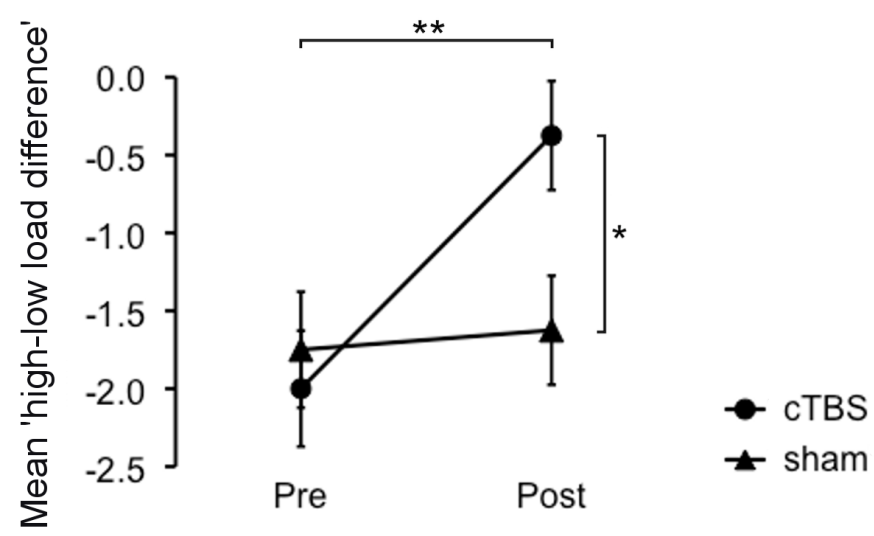


Figure 4

\section{Visual fixations on screen sides}

Low load

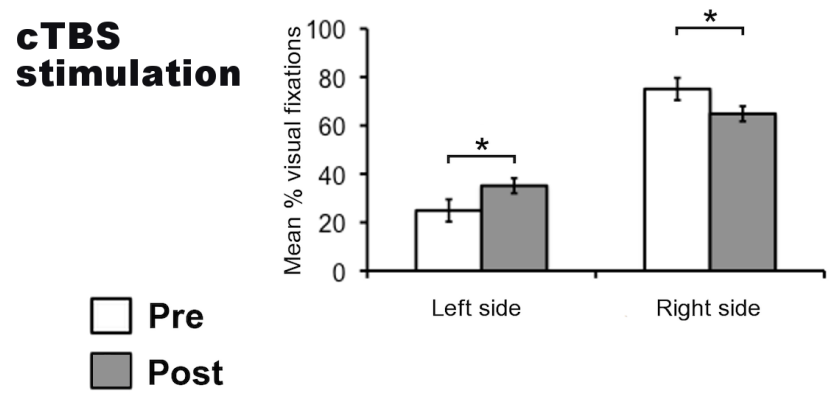

Low load

sham

stimulation

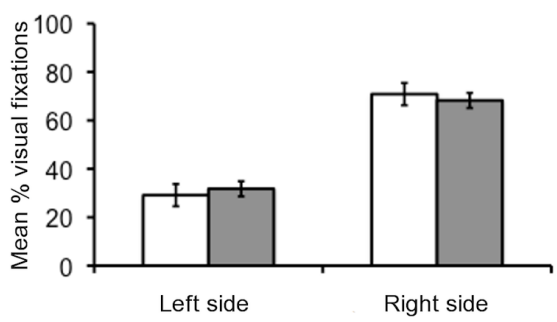

High load

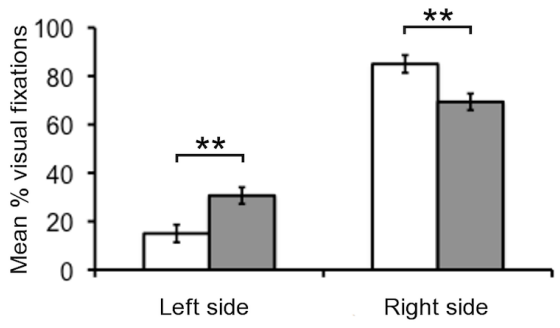

High load

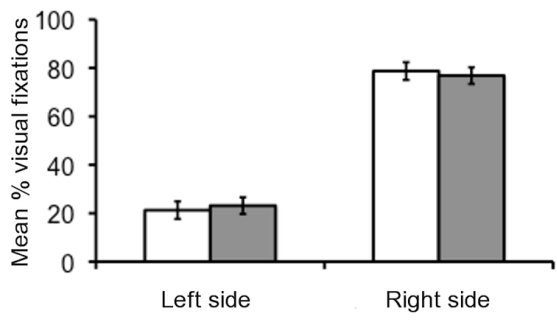


Figure 5

\section{CTBS}

\section{stimulation}
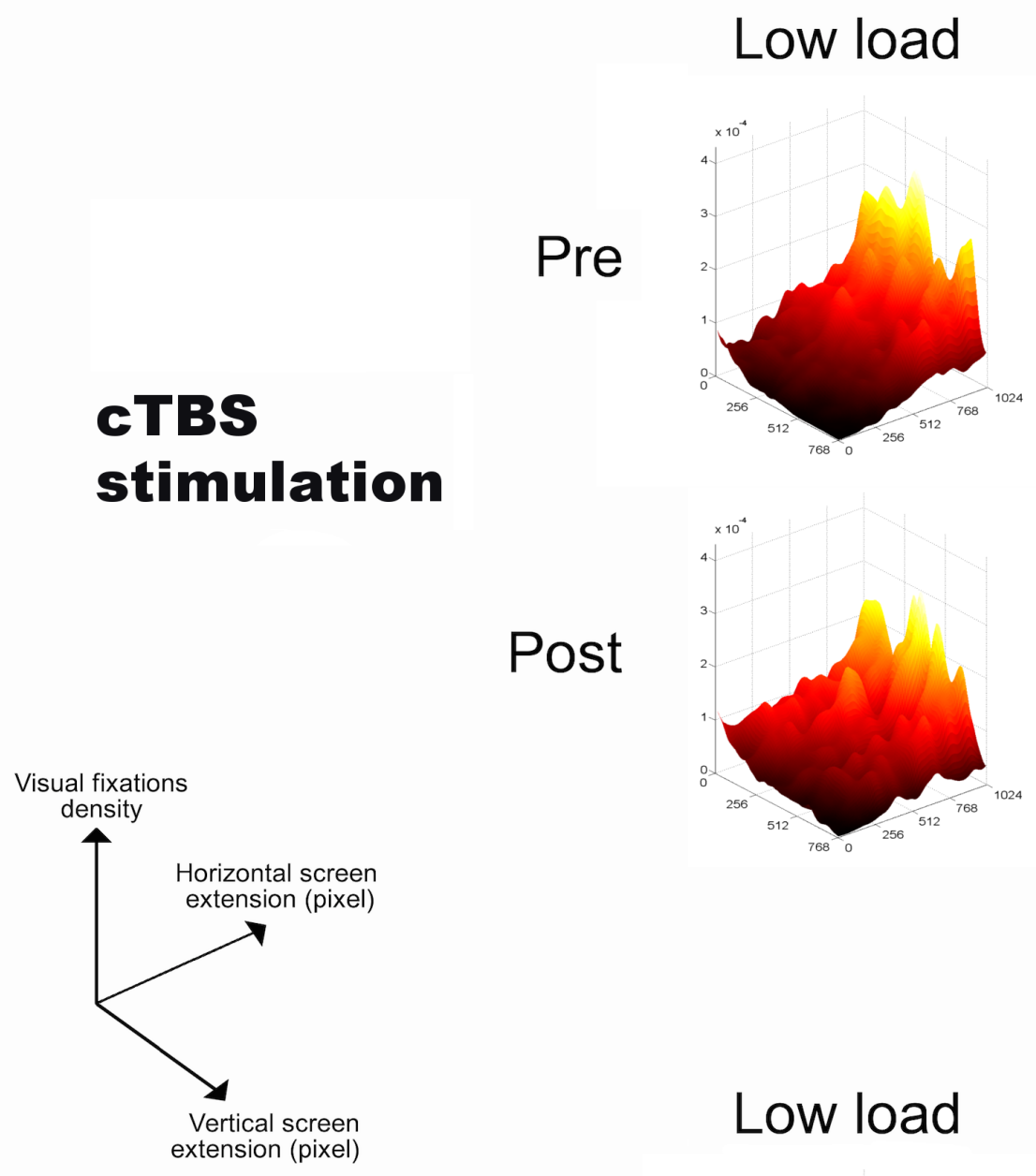

High load

\section{sham} stimulation

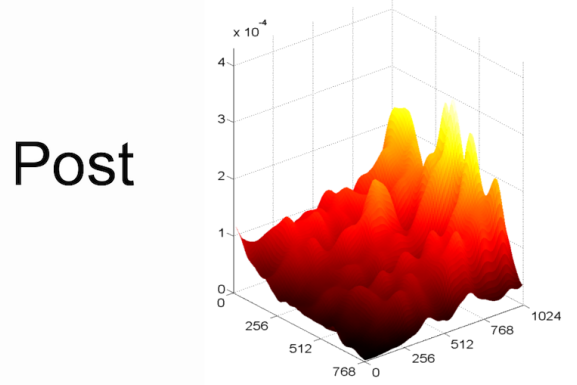

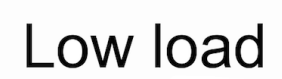
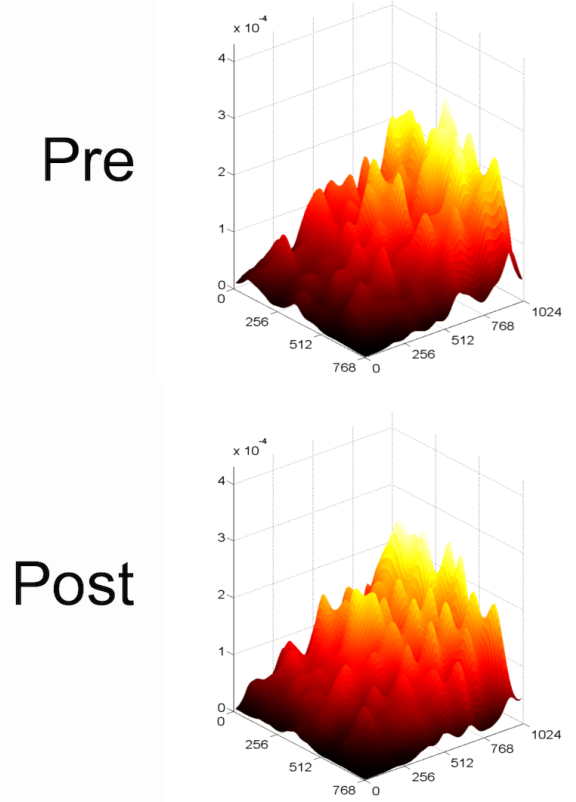
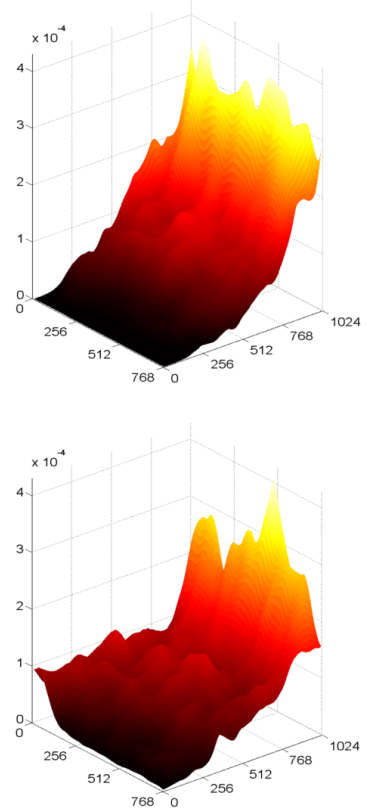

High load
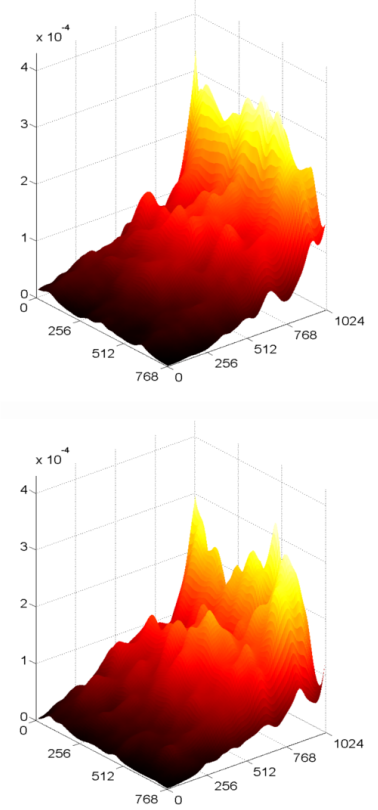
Figure 6

Paper-pencil cancellation tasks

Low load

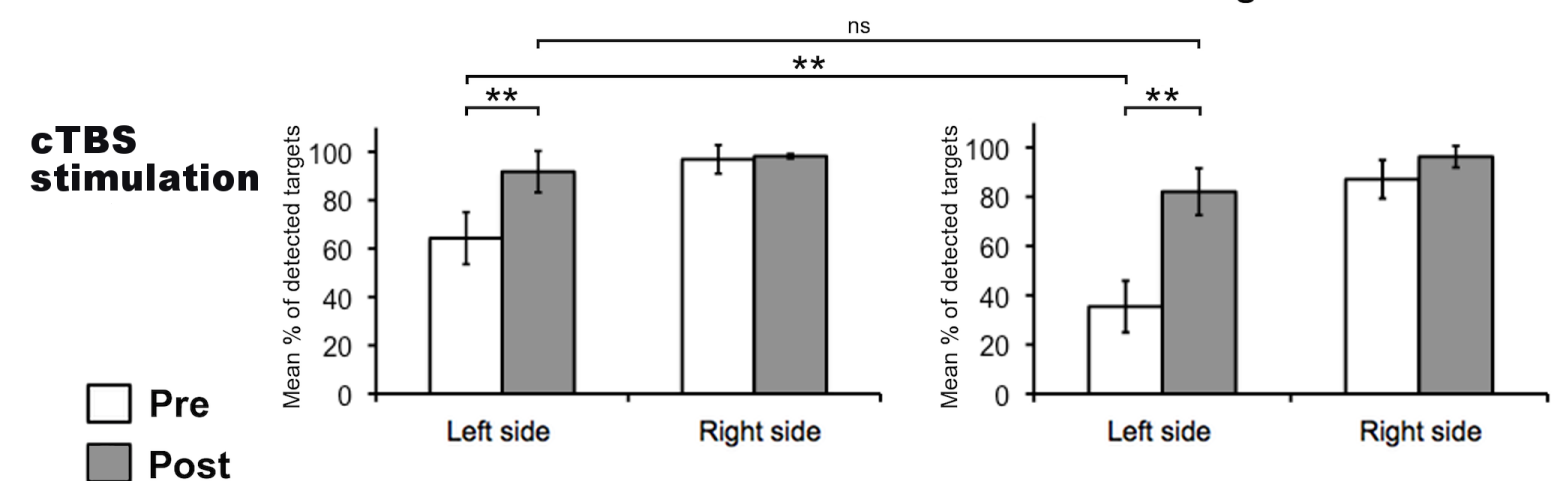

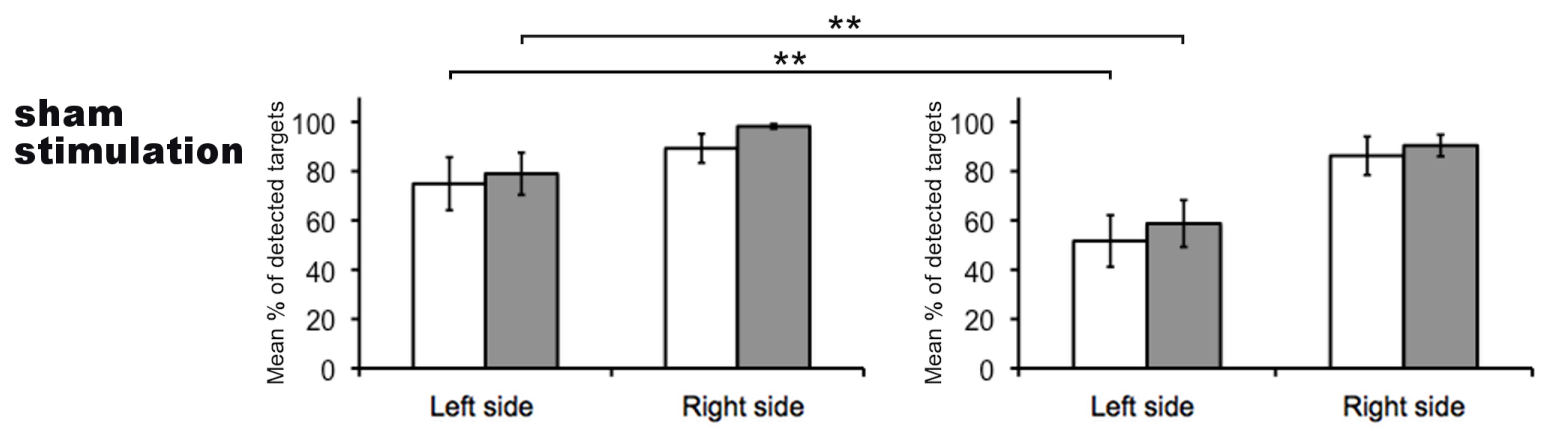

